\title{
Real-world Evaluation of a Novel Technology-enabled Capnometry- assisted Breathing Therapy for Panic Disorder
}

\author{
Divya K. Madhusudhan', Kore N. Glied', Eugene Nguyen', Jennifer Rose', Dena M. Bravata',2* \\ 1 Crossover Health, San Clemente, CA \\ 2Center for Primary Care and Outcomes Research, Stanford University School of Medicine, Stanford, CA
}

\section{Article Info}

\section{Article Notes}

Received: October 8, 2020

Accepted: November 18, 2020

\section{*Correspondence:}

Dr. Dena M. Bravata, M.D., M.S., Crossover Health, San Clemente, CA; Senior Affiliate, Center for Primary Care and Outcomes Research, Stanford University School of Medicine; 1840 Lexington Av, San Mateo CA; Telephone No: 415-7065829; Email: dbravata@gmail.com.

(c) 2020 Bravata DM. This article is distributed under the terms of the Creative Commons Attribution 4.0 International License.

\section{Keywords:}

Behavioral Health

Occupational Health

Breathing Therapy

Panic Disorder

Post-traumatic Stress Disorder

Real-world Evaluation
Abstract

Introduction: Anxiety disorders which include generalized anxiety disorder, panic disorder (PD), post-traumatic stress disorder, and obsessive-compulsive disorder are common psychiatric condition associated with significant social, occupational, physical, and economic costs. Interventions that target the respiratory dysregulation associated with anxiety disorders have demonstrated considerable therapeutic benefit. However, no such intervention has been evaluated in among PD patients receiving care in an employer-sponsored health center.

Objective: To evaluate the use of a novel, capnometry-assisted breathing therapy on clinical outcomes, engagement, and satisfaction for patients with $\mathrm{PD}$ receiving care in an employer-sponsored health center.

Methods: Prospective analysis of participants using the program between $1 / 1 / 2018$ and 06/30/2020.

Results: Twenty-two participants enrolled. Their average weekly usage remained high throughout the 4-week treatment protocol of 17 minutes twice a day for 28 days (11.6 sessions (SD 3.1) in week one and 10.4 sessions (SD 4.0 ) in week four). Their average Panic Disorder Severity Scale score decreased from 13.3 (SD \pm 3.6 ) to 5.6 (SD \pm 1.8 ), indicating that panic severity decreased from "moderately ill" to "slightly ill." Use of the program was associated with clinically and statistically significant improvements in respiratory markers: average respiratory rate decreased from 14.9 (SD 3.6) to 11.9 (SD 4.7) breaths per minute $(p<0.001)$ and end-tidal $\mathrm{pCO}_{2}$ increased from $37.2 \mathrm{mmHg}$ (SD 4.2) to $39.1 \mathrm{mmHg}$ (SD 3.6) ( $p<0.001)$. Engagement was associated with decline in utilizations of behavioral health services at employer-sponsored primary care center clinic after the intervention.

Conclusion: The clinical improvements observed in this pilot suggest that capnometry-assisted breathing therapy is a valuable tool for patients suffering from panic-associated symptoms.

\section{Background / Introduction}

Anxiety disorders which include generalized anxiety disorder, PD, PTSD, and obsessive-compulsive disorder are common psychiatric conditions associated with significant social, occupational, physical, and economic costs ${ }^{1,2}$. Approximately 27 million US adults experience panic attacks annually with over 6.7 million suffering from PD and 8 million from PTSD ${ }^{3,4}$. Anxiety disorders are associated with poor workplace productivity and short and long term disabilities ${ }^{5,6}$ accounting for approximately $\$ 45$ billion in annual health care spending ${ }^{7}$. While these conditions are commonly treated with pharmacotherapy and/or psychotherapy, not all patients respond or achieve full recovery with these modalities ${ }^{8}$. 
Growing evidence suggests that respiratory dysregulation is a central feature of PD. Klein and colleagues' 'suffocation false alarm' hypotheses regarding carbon dioxide hypersensitivity set the stage for the examination of the respiratory irregularities associated with panic disorder and related conditions ${ }^{9,10}$. In the case of panic disorder, chronic hyperventilation, lower end tidal $\mathrm{CO}_{2}$ levels, respiratory rate and tidal volume irregularities, sighing, and breath-holding are characteristic features ${ }^{11}$. The literature on respiratory dysregulation in PTSD is less extensive. However, a comparative analysis of PD and PTSD found that subjects with PTSD also showed abnormal breathing patterns (high sigh rate, more abdominal breathing, and only slightly less hypocapnia than the PD group ${ }^{12}$. Additional evidence linking underlying respiratory pathophysiology of PD and PTSD includes the bidirectional relationship between the two disorders ${ }^{13}$ and reactivity to $\mathrm{CO}_{2}$ challenge testing ${ }^{14}$. Again, the literature for $\mathrm{CO}_{2}$ challenge is more substantial for $\mathrm{PD}$, but an emerging body of evidence for PTSD points to $\mathrm{CO}_{2}$ reactivity as a risk factor for development of PTSD in military subjects ${ }^{15}$ and is characteristic in patients with PTSD ${ }^{16}$. In the trial of PTSD by Kellner and colleagues, they found that patients with PTSD were not reactive to placebo gas exposure whereas some subjects experienced post-traumatic flashbacks in addition to panic symptoms when exposed to a single inhalation of $35 \% \mathrm{CO}_{2}{ }^{16}$.

Interventions that specifically target these respiratory pathways have demonstrated considerable therapeutic benefit among these patient populations ${ }^{17-19}$. Anxiety disorders can trigger the fight-or-flight response, which stimulates the sympathetic nervous system to increase heart rate and blood pressure and leads to over-breathing among other stress reactions. Breathing exercises can reduce the body's reaction to stress and anxiety by mediating this relationship with the sympathetic nervous system. Specifically, breathing exercises are associated with decreased heart rate, respiratory rate (RR), and blood pressure ${ }^{20}$. Recognition is growing that treatments that effectively treat panic-related conditions (psychopharmacological as well as psychotherapeutic) also reduce $\mathrm{CO}_{2}$ sensitivity ${ }^{11}$. Berenz and colleagues suggest treatment implications as follows: "Given the association between PTSD and subsequent PD, particularly among men, clinicians may consider supplementing PTSD treatment with panic specific interventions"13.

A novel technology called Freespira was developed to train participants to reduce their RR and increase their exhaled end-tidal $\mathrm{pCO}_{2}$ levels through structured breathing exercises. This technology has not been previously evaluated in an employer-sponsored setting. However, given its demonstrated effectiveness in managing PD, PTSD, among other common anxiety-associated disorders, ${ }^{18,21}$ this intervention holds promise for reducing the morbidity and associated cost among employee populations. The purpose of this pilot was to evaluate Freespira among a population of adult employees with PD receiving care in an employer-sponsored health center. Specifically, we sought to determine whether completing a 28-day intervention with Freespira would: 1) Reduce symptom severity as measured by condition-specific scales (i.e., Panic Disorder Severity Scale (PDSS) for panic disorder, Post Traumatic Stress Disorder checklist (PCL 5) for PTSD, Patient Health Questionnaire (PHQ-9) for depression, and Generalized Anxiety Disorder questionnaire (GAD-7) for generalized anxiety disorder; 2) Improve respiratory parameters as measured by end-tidal $\mathrm{pCO}_{2}$ and RR; and 3) Provide a positive experience as measured by engagement rates.

\section{Materials and Methods}

\section{Participants}

Eligible participants were adult Comcast employees with PD receiving care in an employer-sponsored Crossover health center located in Midtown, New York City between $1 / 1 / 2018$ and $6 / 30 / 2020$. Three training seminars were conducted with primary care and behavioral health providers to provide education about the intervention, inclusion criteria, and the referral process. These seminars were attended by more than 50 providers and clinic staff who made 45 referrals. Patients who were referred to Freespira and expressed interest in the program were sent an assessment packet via secure email and given the Freespira enrollment phone number. If a patient did not complete the assessment packet or call Freespira, there was no additional follow up. Participants were considered ineligible for inclusion if they were pregnant or diagnosed with obesity hyperventilation syndrome. Participants did not incur incremental costs or receive any incentives for study participation.

\section{Intervention}

Freespira is a patented, FDA-cleared digital therapeutic solution for the treatment of PD, PTSD, agoraphobia, and other disorders where panic attacks are common. The intervention trains patients, through physiological feedback, to increase their end-tidal $\mathrm{pCO}_{2}$ by reducing their RR and respiratory volume. The capnometry-assisted breathing treatment is delivered via a hand-held respiratory sensor, nasal sampling cannula, and software that guides the user through a structured training session, and a dedicated coach providing weekly support. The sensor measures end-tidal $\mathrm{pCO}_{2}$ and RR. Data from this sensor are transmitted via Bluetooth to a tablet preloaded with the Freespira app. The app provides real-time feedback to the user based on their RR and end-tidal $\mathrm{pCO}_{2}$ in accordance with a structured protocol to regulate their breathing. The 
home-based protocol requires two 17-minute breathing/ training sessions per day for 28 days.

Each therapy session consist of three parts: 1) A baseline period (baseline stage), during which participants sit quietly with their eyes closed for two minutes. 2) A 10-minuted paced breathing period (pacing stage) during which participants are instructed to breathe in sync with a series of rising and falling audio tones while viewing their exhaled end-tidal $\mathrm{pCO}_{2}$ level. The tones were set for 13,11 , 9 or 6 breaths per minute, representing each progressive week of the program. By reducing RR and inspired volume, participants learn to raise their exhaled $\mathrm{pCO}_{2}$ into the target zone (around $40 \mathrm{~mm} \mathrm{Hg}$ ) while engaging in paced breathing. And 3) a 5-minute breathing period without pacing tones during which participants are asked to maintain their previously paced RR and end-tidal $\mathrm{pCO}_{2}$ level using the feedback device only, without pacing tones (transition stage). The mobile app showed participants their current end-tidal $\mathrm{pCO}_{2}$ level, target baseline endtidal $\mathrm{pCO}_{2}$ level (37-40 $\mathrm{mm} \mathrm{Hg}$ ), current $\mathrm{RR}$, and target RR (12-16). The repetitive at-home practice also teaches participants to be more aware of episodes of dysfunctional breathing and provides self-management skills to improve breathing.

Session records are uploaded to a secure server following each session and are reviewed by a clinicalspecialist who meets weekly (via video) with the participant to review progress and provide tips and encouragement. If participation decreased or stopped, the patient care specialist contacted the participant to discuss any challenges and offer support or encouragement to schedule an appointment with a behavioral health provider in their employer-sponsored health center as appropriate. If a participant was in crisis (e.g., endorsed suicidal symptoms), the patient care specialist referred the participant to a nearby emergency room for clearance before having the participant continue the intervention. At the end of the intervention, participants returned the device to Palo Alto Health Sciences, Inc (the developer of Freespira).

\section{Data Collection}

Two types of clinical outcomes were measured at baseline and at the end of the intervention: 1) Those related to behavioral health symptoms and 2) those related to respiratory measures. The behavioral health outcome measures were assessed through changes in three clinician-scored, validated metrics. The Panic Disorder Severity Scale (PDSS) for panic disorder severity ${ }^{22}$ (a $40 \%$ or greater reduction in PDSS score was considered clinically significant and remission was defined as a score of $\leq 5$ on the PDSS ${ }^{23,24}$ ), the PTSD Checklist survey (PCL 5) for $\mathrm{PTSD}^{17}$ (a change of 10 or more points was considered clinically significant $\left.{ }^{25,26}\right)$, Patient Health Questionnaire (PHQ 9) ${ }^{27}$ for depression, and Generalized Anxiety Disorder questionnaire (GAD 7) ${ }^{28}$ for generalized anxiety disorder.

The change in average $\mathrm{RR}$ and end-tidal $\mathrm{pCO}_{2}$ during the baseline stage were the key respiratory outcomes of interest as they reflect the ability of the participant to control their breathing without an intervention. The normal RR for an adult at rest ranges from 12 to 16 breaths per minute ${ }^{29}$. The target range end-tidal $\mathrm{pCO}_{2}$, was 37 to $40 \mathrm{~mm} \mathrm{Hg}$. Notably, increases in end-tidal $\mathrm{pCO}_{2}$ levels have been correlated with decreased PDSS severity ${ }^{17}$.

We sought to evaluate the extent to which participants principally received care through Crossover or were utilizing community-based healthcare services during the pilot period. Crossover defines patients as being highly engaged more than $75 \%$ of their health services were delivered through a Crossover provider. Health services utilization and medication usage were abstracted from the Crossover health record for each participant.

Patient satisfaction was measured on a 5-point Likert Scale administered via email at the end of the treatment asking "Did the Freespira treatment help you?" and a Net Promoter Score (NPS) question "How likely are you to recommend this treatment to a friend or family member who suffers from panic attack?"

Baseline data were collected before starting the Freespira intervention and upon completion of the 28-day program. Given that the intervention has been evaluated for safety and efficacy in other settings, and all data used in this analysis are routinely collected on all patients cared for by clinicians in the employer-sponsored clinic and among users of the Freespira device, this study was considered exempt from IRB approval.

\section{Statistical Methods}

We evaluated the average of each behavioral health scale at the start and and end of the intervention. We used linear regression to evaluate the rate of change of RR and exhaled $\mathrm{CO}_{2}$ as a function of the number of training sessions per participant. We expected and tested for a decrease in RR and increase in exhaled end-tidal $\mathrm{CO}_{2}$. Homoscedasticity and mean average of residuals were checked to ensure non-violation of regression assumptions. All statistical analyses were conducted with Python 3.8, pandasv1.05, and Statsmodels v0.12.0.

\section{Results}

\section{Participant Characteristics}

Table 1 presents demographic and clinical characteristics of the 22 participants who enrolled in the program and completed at least one breathing session. Participants' average age was 35 years (SD 6.8). They 
Table 1. Participant Characteristics and Program Participation

\begin{tabular}{|c|c|}
\hline Characteristic & Mean (SD) or $\mathbf{N}(\%)$ \\
\hline Age (years) & $34(6.8)$ \\
\hline Female & $16(72.7 \%)$ \\
\hline \multicolumn{2}{|c|}{ Diagnoses and common comorbidities } \\
\hline Panic Symptoms & 19 \\
\hline Generalized Anxiety Disorder & 18 \\
\hline PD & 8 \\
\hline Obsessive-Compulsive Disorder & 3 \\
\hline PTSD & 2 \\
\hline Major Depressive Disorder & 2 \\
\hline Social Phobias & 2 \\
\hline Agoraphobia & 1 \\
\hline \multicolumn{2}{|c|}{$\begin{array}{l}\text { Crossover Health Services Utilization (At } \\
\text { least one visit) }\end{array}$} \\
\hline Behavioral Health & 20 \\
\hline Primary Care & 18 \\
\hline Preventive Care Visit & 15 \\
\hline Optometry & 12 \\
\hline Chiropractic & 10 \\
\hline Physical Therapy & 8 \\
\hline Health Coaching & 4 \\
\hline Acupuncture & 4 \\
\hline Massage & 2 \\
\hline
\end{tabular}

were mostly female (73\%). Five participants had a principal diagnosis of PD, two had PTSD, and 19 had panic symptoms including tachycardia, palpitations, generalized hyperhidrosis, or emotional lability (note: participants could have both PTSD and some panic symptoms). Additionally, 19 participants (86\%) were diagnosed with a comorbid behavioral health condition such as depression or anxiety. Seven participants were prescribed psychoactive medications during the pilot including common antidepressants and anxiolytics.

\section{Utilization of Services in the Employer-sponsored Health Center}

Sixteen (73\%) participants were considered highly engaged members of their employer-sponsored health center with no community utilization for behavioral health issues during the evaluation period. This was significantly higher than the rate of engagement for the overall employer population (14\%). The number of behavioral health visits at the employer-sponsored clinic for the 22 participants during the 30 days before starting the Freespira intervention was 52 . This increased to 57 visits during the intervention and fell to 44 visits during the 30 days after the intervention.

\section{Engagement in the Intervention}

The Freespira protocol recommends two 17-minute sessions per day for 28 days (56 total breathing sessions). On an average, pilot participants completed 44.2 (SD 26.0) breathing sessions over the course of four weeks (an 83\% adherence rate). During the first week, on average, participants completed 267 sessions with an average weekly usage of 11.6 sessions (SD 3.1). During the final week of the pilot, participants had only a slightly lower engagement rate with an average of 10.4 (SD 4.0) at week four.

\section{Clinical Outcomes}

\section{Behavioral Health Outcomes}

Among the 22 participants, 18 provided both their initial and final PDSS assessments. Their initial PDSS score decreased on average from 13.3 (SD \pm 3.6 ) to $5.6(\mathrm{SD} \pm$ 1.8), an average decrease of 7.2. Panic severity decreased from "moderately ill" to "slightly ill". Further, 12 out of 18 participants $(67 \%)$ had a clinically significant $(40 \%$ or greater) decrease in their PDSS score.

Two participants with a diagnosis of PTSD provided both their initial and final PCL 5 scores. Their initial PCL 5 score decreased from $41.0(\mathrm{SD} \pm 13.1$ ) to 27.5 ( $S D \pm 6.4$ ), an average decrease of 13.5 .

Eleven participants provided both initial and final PHQ 9 scores for depression. Their initial score decreased from 11.1 (SD \pm 6.3 ) to 9.1 (SD \pm 7.1 ) progressing from "moderate depression" to "mild depression." The four individuals who had a baseline PHQ $9 \geq 10$ (moderately severe) had an average initial PHQ9 score of 15.8 (SD \pm 3.4 ) that declined to $15.0(\mathrm{SD} \pm 4.2)$ indicating no meaningful change in comorbidity.

Eleven participants provided both initial and final GAD 7 scores for anxiety. Their average initial score decreased from $8.6(\mathrm{SD} \pm 6.2)$ to $7.5(\mathrm{SD} \pm 6.6)$ at the end of the intervention indicating 'moderate anxiety'. Seven individuals who had a baseline GAD $7 \geq 10$ had an average initial score that declined from 15.1 ( $S D \pm 2.9$ ) to $12.3(\mathrm{SD} \pm 7.0)$. This improvement was not statistically significant.

\section{Respiratory outcomes}

Among the 22 participants, their baseline RR decreased from 14.9 (SD 3.6) to 11.9 (SD 4.7) breaths per minute (Table 2). The regression results (Figure 1 and Table 3a) show that this decline in RR was significantly associated with the number of sessions a participant completed $(\mathrm{p}<0.001)$.

Figure 2 shows the significant improvement in endtidal $\mathrm{pCO}_{2}$ over the course of the intervention. The average end-tidal $\mathrm{pCO}_{2}$ in the baseline stage increased from $37.2 \mathrm{mmHg}$ (SD 4.2) at week one to $39.1 \mathrm{mmHg}$ (SD 3.6) at week four. The regression analysis showed (Table $3 \mathrm{~b}$ ) that the end-tidal $\mathrm{pCO}_{2}$ increase was significantly associated with the number of sessions completed $(\mathrm{p}<0.001)$. On 


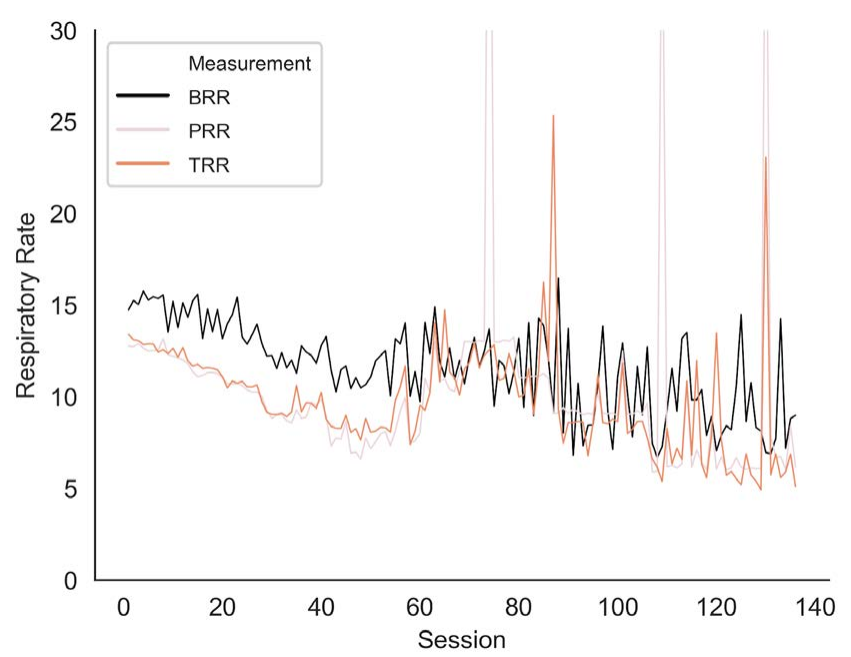

Figure 1: Average respiratory rate over time

Table 2: Respiratory Rate Changes by Phase Over Time

\begin{tabular}{|l|c|c|c|}
\hline & Baseline RR (SD) & Pacing RR (SD) & Transition RR (SD) \\
\hline Week 1 & $14.89(3.59)$ & $12.60(0.89)$ & $12.75(1.93)$ \\
\hline Week 2 & $13.94(3.59)$ & $11.10(1.26)$ & $11.42(2.16)$ \\
\hline Week 3 & $12.63(4.72)$ & $9.41(2.01)$ & $9.63(2.61)$ \\
\hline Week 4 & $11.87(4.71)$ & $7.82(2.69)$ & $8.57(3.43)$ \\
\hline
\end{tabular}

Table 3a. Regression Results: Respiratory Rate vs. Sessions

\begin{tabular}{|l|c|c|c|c|c|}
\hline & $\mathbf{b}$ & $\mathbf{b ~ C l}$ & Std. Error & Intercept & $\mathbf{p}$ \\
\hline Baseline RR & -.0574 & $-.067, .048$ & .005 & 14.91 & $<.001$ \\
\hline Pacing RR & -.0420 & $-.049, .035$ & .004 & 11.62 & $<.001$ \\
\hline Transition RR & -.0498 & $-.057, .043$ & .004 & 12.09 & $<.001$ \\
\hline
\end{tabular}

Table 3b. Regression Results: End-Tidal $\mathrm{CO}_{2}$ vs. Sessions

\begin{tabular}{|l|c|c|c|c|c|}
\hline & $\mathbf{b}$ & $\mathbf{b ~ C l}$ & $\begin{array}{c}\text { Std. } \\
\text { Error }\end{array}$ & Intercept & $\mathbf{p}$ \\
\hline Baseline End-tidal $\mathrm{CO}_{2}$ & .0501 & $.041-.059 .004$ & 37.11 & $<.001$ \\
\hline Pacing End-tidal $\mathrm{CO}_{2}$ & .0520 & $.044-.060 .004$ & 37.06 & $<.001$ \\
\hline Transition End-tidal $\mathrm{CO}_{2}$ & .0528 & $.050-.068$ & .004 & 36.79 & $<.001$ \\
\hline
\end{tabular}

average, for every completed session, end-tidal $\mathrm{pCO}_{2}$ in the baseline phase increased by approximately $0.05 \mathrm{mmHg}$ $(\mathrm{p}<0.001)$. We observed that end-tidal $\mathrm{pCO}_{2}$ increased in parallel during paced and transition stages suggesting that participants were able to maintain control over their breathing pattern without guiding tones.

\section{Participant Satisfaction}

The response rate on the patient satisfaction survey and NPS was low (only 7 (27\%) participants). Six participants reported that the intervention helped them or significantly helped them and that they would recommend it to a friend or family member with panic attacks.

\section{Discussion}

Overall, the use of Freespira was associated with clinically and statistically significant improvements in

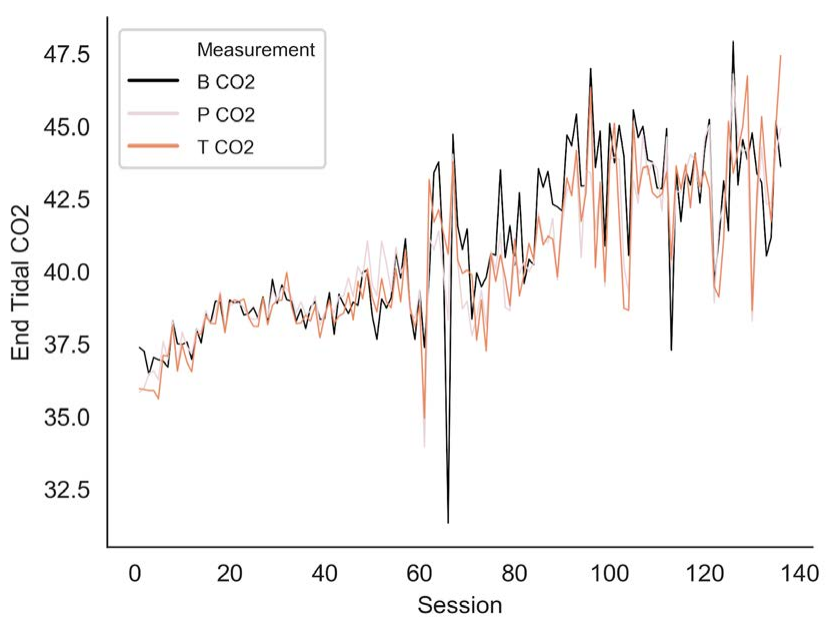

Figure 2a: Average end-tidal $\mathrm{pCO}_{2}$ over time

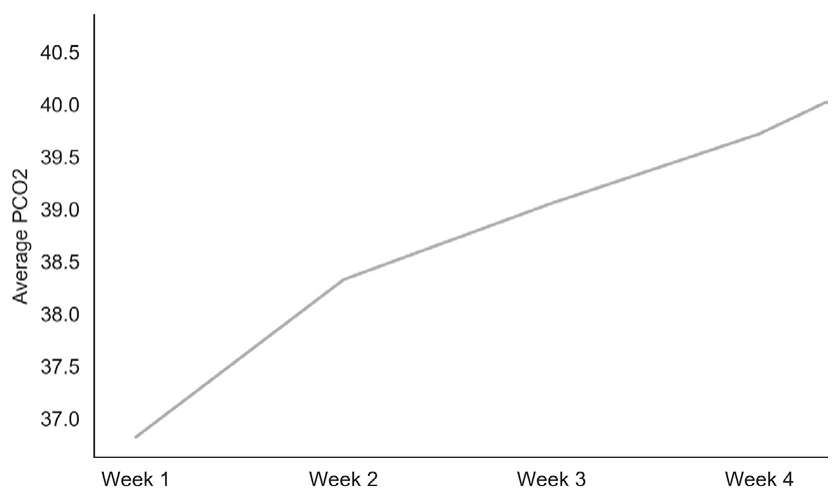

Figure $\mathbf{2 b}$ : Average end-tidal $\mathrm{pCO}_{2}$ in the 'baseline phase' over time

key clinical outcomes among a population of employees receiving care for $\mathrm{PD}$ in an employer-sponsored health center. This real-world evaluation of the capnometry-based intervention had four key findings.

First, $82 \%$ of participants experienced significant improvements in PD severity and increased end-tidal $\mathrm{pCO}_{2}$ levels. Improvements in end-tidal $\mathrm{pCO}_{2}$ levels and $\mathrm{RR}$ were significantly associated with the number of sessions a participant completed. Tolin et al. demonstrated durable benefits of Freespira at 12 months of follow up after a 4-week intervention identical to the intervention used in this pilot. ${ }^{18}$ Thus, although we did not evaluate clinical outcomes beyond four weeks, it is likely that benefits continued after treatment discontinuation. Although the demographics of the enrolled participants skewed female $(73 \%)$, we believe the results are generalizable to employee populations given that women are more than twice as likely to suffer from PD than men ${ }^{31,32}$. Women have been reported to be more likely to seek psychotherapy than men $^{33,34}$ - so, a technology like Freespira might be a novel means of getting care to men with PD who are reluctant to engage in psychotherapy. 
Second, $86 \%$ of participants were diagnosed with a comorbid behavioral health condition such as depression or anxiety, although depression was mild in all but four participants. The relationship between PD and generalized anxiety and depression has been previously documented ${ }^{35,36}$ with $98 \%$ of PD patients developing at least one comorbid psychiatric disorder ${ }^{37}$. Given the recent negative impact of COVID-19 pandemic on the mental health of adults in the $\mathrm{US}^{38}$, future studies of Freespira should evaluate populations with more significant comorbid depression and anxiety to characterize populations most likely to benefit from either Freespira alone or delivered in combination with either medication or psychotherapy. Moreover, given the hesitance of many patients to seek care in person due to concerns over COVID-19 transmission, digital mental health solutions can be a valuable tool for getting care to people outside of the clinical setting and helping prioritize in-person or videobased care to higher acuity populations ${ }^{39}$.

Third, participants were highly engaged with the Freespira program over the course of the 28-day intervention with little drop off even in the final week of the therapy. Although relatively few participants provided satisfaction or NPS data, the high-level of engagement suggests that the program was tolerable to patients with PD. These data are consistent with prior findings: A previously published trial ${ }^{40}$ of the Freespira intervention recorded high satisfaction rates $(84 \%)$ and adherence $(88 \%)$ in a panic disorder population, while data available from the Freespira FDA clearance records for PTSD showed satisfaction rates of $84 \%$ and adherence rates of $77 \%{ }^{41}$. The positive adherence and satisfaction rates along with a minimal side effect profile reported by the FDA show promise in expanding treatment options for PTSD, whose most effective psychotherapies, namely trauma-focus methods, differentially show high dropout rates ${ }^{40}$.

Fourth, 73\% participants were considered highly engaged members of their employer-sponsored health center with no community utilization for behavioral health issues during the evaluation period. The decline in behavioral health visits after the intervention (from 52 to 44 ) is a promising finding that suggests that controlling panic and related symptoms improved overall need for health services and may result in decreased associated health related spending. This warrants a larger trial specifically designed to evaluate key economic outcomes associated with Freespira. A recent study of a Highmark population using Freespira showed similar results and was associated with reduced medication cost and fewer specialist and emergency department visits ${ }^{21}$. Engaging in evidence-based digital health intervention such as Freespira may improve access to care and reduce provider burden at employersponsor care centers. The demand for behavioral health care is increasing and access to in-person behavioral health providers is often delayed with an average wait time of 25 days for a first visit in the community ${ }^{42}$. Moreover, potential benefits of direct access to an evidence-based digital health solution include greater anonymity and confidentiality lowering concerns regarding any stigma associated with $\mathrm{PD}^{43}$. Use of the Freespira program could provide some care for individuals for whom access to in-person services may be limited or delayed. Notably, given that most of the participants in this pilot were also receiving behavioral health care at Crossover, we were not able to compare the clinical outcomes of participants receiving just Freespira versus Freespira plus in-person psychotherapy. This should be a priority for future evaluations.

Regarding possible mechanisms of action associated with clinical benefit of this intervention, Freespira targets respiratory rate and $\mathrm{CO}_{2}$ levels in conditions known to be carbon dioxide sensitive. As in prior studies ${ }^{40,44}$, the current study population showed significant normalization of both respiratory rate and end tidal $\mathrm{CO}_{2}$ levels across the course of the trial. Given the complexity of the pathophysiology of PD, it is unlikely that a unitary mechanism of action is responsible for the treatment benefits, but Freespira is unique among current treatment options in directly addressing carbon dioxide physiology. It is noteworthy that a randomized wait-list control trial of a paced breathing intervention that did not include feedback of exhaled $\mathrm{CO}_{2}$ showed no benefit in reducing panic, anxiety, or hyperventilation symptoms ${ }^{42}$. Additional research ${ }^{45}$ on potential mechanisms of action for this intervention support conclusions that normalization of $\mathrm{CO}_{2}$ levels is causily related to symptom improvement. Development of perceived control was also recognized as an additional and important mechanism of change inherent in both cognitive and respiratory interventions. In addition to addressing respiratory physiology, the Freespira intervention is aimed at developing and sustaining self-management skills that can be utilized by patients after the conclusion of the intervention.

This study had two important limitations. The relatively small sample size (especially of PTSD patients) limited our ability to evaluate whether the use of Freespira could meaningfully serve as a means of treating employees remotely, without additional in-person care or to understand if clinical improvements in panic symptoms were associated with economic and other key outcomes. PD and PTSD are relatively less common among employee patients than anxiety and adjustment disorder. Given prior published evidence for the effectiveness of Freespira to improve many symptoms of anxiety, we recommend that the inclusion criteria for future evaluations be expanded to include these patients.

Additionally, the pilot did not restrict participants from seeking additional psychotherapy or using medication 
therapy for their mental health while using the Freespira program. We cannot dismiss the possibility of either medications or psychotherapy contributing to clinical improvement. An active control intervention would be required to rule out potential effects of therapy factors.

\section{Conclusions}

The clinical improvements observed in this study suggest that Freespira is a valuable tool for employees suffering from panic associated symptoms. For it to have greater impact both clinically and economically, it should be evaluated on much larger employee and general populations not also receiving in-person care.

\section{Authors' Contributions}

All authors participated in the design, implementation, or analysis of this study. They approved the final draft of the manuscript.

\section{Acknowledgements}

We thank the team at Palo Alto Health Sciences Inc. (the manufacturer of the Freespira device) for their support with this study.

\section{Funding}

This work was fully supported by Comcast NBCUniversal, Crossover Health, and Palo Alto Health Sciences, Inc (the developer of Freespira).

\section{Conflict Of Interests}

All authors were either employees of or consultants to Crossover Health at the time of this work.

\section{References}

1. Walker EA, Katon W, Russo J, et al. Health care costs associated with posttraumatic stress disorder symptoms in women. Arch Gen Psychiatry. 2003; 60(4): 369-374.

2. Smit F, Willemse G, Meulenbeek $P$, et al. Preventing panic disorder: cost-effectiveness analysis alongside a pragmatic randomised trial. Cost Eff Resour Alloc. 2009; 7: 8.

3. Multiple Studies show Freespira eliminates panic attacks in $80 \%$ of patients. 2018.

4. National Center for PTSD. 2019. https://www.ptsd.va.gov/ understand/common/common_adults.asp. Accessed May 20, 2020.

5. Hendriks SM, Spijker J, Licht CM, et al. Disability in anxiety disorders. J Affect Disord. 2014; 166: 227-233.

6. Plaisier I, de Graaf R, de Bruijn J, et al. Depressive and anxiety disorders on-the-job: the importance of job characteristics for good work functioning in persons with depressive and anxiety disorders. Psychiatry Res. 2012; 200(2-3): 382-388.

7. Devane CL, Chiao E, Franklin M, et al. Anxiety disorders in the 21st century: status, challenges, opportunities, and comorbidity with depression. Am J Manag Care. 2005; 11(12 Suppl): S344-353.

8. Caldirola D, Perna G. Toward a personalized therapy for panic disorder: preliminary considerations from a work in progress. Neuropsychiatr Dis Treat. 2019; 15: 1957-1970.
9. Klein DF. False suffocation alarm, spontaneous panic, and related conditions, An integrative hypothesis. Arch Gen Psych. 1993; 50: 306-17.

10. Preter M. Lifelong opioidergic vulnerability through early life separatin: A recent extension of the false suffocation alarm theory of panic disorder, in Nardi AE, Freire RCR (eds.) Panic Disorder: Neurobiological and Treatment Aspects, Springer International Publishing, Switzerland. 2016.

11. Perna G, Iannone G, Torti T, et al. Panic Disorder: Is it really a mental disorder? From body functions to the homeostatic brain, in Nardi AE, Freire RCR (eds.) Panic Disorder: Neurobiological and Treatment Aspects, Springer International Publishing, Switzerland. 2016.

12. Blechert J, Michael T, Grossman P, et al. Autonomic and respiratory characteristics of post-traumatic stress disorder and panic disorder. Psychosomatic Med. 2007; 69: 935-943.

13. Berenz EC, York TP, Bing-Canar H, et al. Time course of panic disorder and posttraumatic stress disorder onsets. Social Psych and Psych Epi. 2019; 54: 639-647.

14. Amaral JMX, Spadaro PTM, Pereirya VM, et al. The carbon dioxide challenge test in panic disorder: a systematic review of preclinical and clinical research. Revista Brasileira de Psiquiatria. 2013; 35: 318-331.

15. Telch MJ, Rosenfield D, Pai A. Emotional reactivity to a single inhalation of $35 \%$ carbon dioxide and its association with later symptoms of posttraumatic stress disorder and anxiety in soldiers deployed to Iraq. Arch Gen Psych. 2012; 60(11): 1161-1168.

16. Kellner M, Muhtz C, Nowack S, et al. Effects of 35\% carbon dioxide (CO2)) inhalation in patients with post-traumatic stress disorder (PTSD): A double-blind, randomized, placebo-controlled, cross-over trial. J Psych Res. 2018; 96: 260-264.

17. Meuret AE, Wilhelm FH, Ritz T, et al. Feedback of end-tidal pCO2 as a therapeutic approach for panic disorder. J Psychiatr Res. 2008; 42(7): 560-568.

18. Tolin DF, McGrath PB, Hale LR, et al. A Multisite Benchmarking Trial of Capnometry Guided Respiratory Intervention for Panic Disorder in Naturalistic Treatment Settings. Appl Psychophysiol Biofeedback. 2017; 42(1): 51-58.

19. Meuret AE, Rosenfield D, Hofmann SG, et al. Changes in respiration mediate changes in fear of bodily sensations in panic disorder. J Psychiatr Res. 2009; 43(6): 634-641.

20. Wilhelm FH, Trabert W, Roth WT. Physiologic instability in panic disorder and generalized anxiety disorder. Biol Psychiatry. 2001; 49(7): 596-605.

21. Kaplan A, Mannarino AP, Nickell PV. Evaluating the Impact of Freespira on Panic Disorder Patients' Health Outcomes and Healthcare Costs within the Allegheny Health Network. Appl Psychophysiol Biofeedback. 2020; 45(3): 175-181.

22. Shear MK, Brown TA, Barlow DH, et al. Multicenter collaborative panic disorder severity scale. Am J Psychiatry. 1997; 154(11): 1571-1575.

23. Furukawa TA, Katherine Shear M, Barlow DH, et al. Evidence-based guidelines for interpretation of the Panic Disorder Severity Scale. Depress Anxiety. 2009; 26(10): 922-929.

24. Davies CD, McGrath PB, Hale LR, et al. Mediators of Change in Capnometry Guided Respiratory Intervention for Panic Disorder. Appl Psychophysiol Biofeedback. 2019; 44(2): 97-102.

25. Blevins CA, Weathers FW, Davis MT, et al. The Posttraumatic Stress Disorder Checklist for DSM-5 (PCL-5): Development and Initial Psychometric Evaluation. J Trauma Stress. 2015; 28(6): 489-498.

26. Bovin MJ, Marx BP, Weathers FW, et al. Psychometric properties of the PTSD Checklist for Diagnostic and Statistical Manual of Mental Disorders-Fifth Edition (PCL-5) in veterans. Psychol Assess. 2016; 28(11): 1379-1391. 
27. Kroenke K, Spitzer RL, Williams JB. The PHQ-9: validity of a brief depression severity measure. J Gen Intern Med. 2001; 16(9): 606-613.

28. Lowe B, Decker O, Muller S, et al. Validation and standardization of the Generalized Anxiety Disorder Screener (GAD-7) in the general population. Med Care. 2008; 46(3): 266-274.

29. John Hopkins Medicine: Vital Signs (Body Temperature, Pulse Rate, Respiration Rate, Blood Pressure). 2020; https://www. hopkinsmedicine.org/health/conditions-and-diseases/vital-signsbody-temperature-pulse-rate-respiration-rate-blood-pressure : : text=When\%20checking\%20respiration\%2C\%20it\%20is,to\%20 16\%20breaths\%20per\%20minute. Accessed May 20, 2020.

30. Bischoff T, Anderson SR, Heafner J, et al. Establishment of a Reliable Change Index for the GAD-7. Psych Community \& Health. 2019; 8(1): 176-187.

31. McLean CP, Asnaani A, Litz BT, et al. Gender differences in anxiety disorders: prevalence, course of illness, comorbidity and burden of illness. J Psychiatr Res. 2011; 45(8): 1027-1035.

32. Tolin DF, Foa EB. Sex differences in trauma and posttraumatic stress disorder: a quantitative review of 25 years of research. Psychol Bull. 2006; 132(6): 959-992.

33. Albizu-Garcia CE, Alegria M, Freeman D, et al. Gender and health services use for a mental health problem. Soc Sci Med. 2001; 53(7): 865-878.

34. Sagar-Ouriaghli I, Godfrey E, Bridge L, et al. Improving Mental Health Service Utilization Among Men: A Systematic Review and Synthesis of Behavior Change Techniques Within Interventions Targeting HelpSeeking. Am J Mens Health. 2019; 13(3): 1557988319857009.

35. Gorman JM, Coplan JD. Comorbidity of depression and panic disorder. J Clin Psychiatry. 1996; 57 Suppl 10: 34-41; discussion 42-33.
36. Lesser IM, Rubin RT, Pecknold JC, et al. Secondary depression in panic disorder and agoraphobia. I. Frequency, severity, and response to treatment. Arch Gen Psychiatry. 1988; 45(5): 437-443.

37. Tilli V, Suominen K, Karlsson H. Panic disorder in primary care: comorbid psychiatric disorders and their persistence. Scand J Prim Health Care. 2012; 30(4): 247-253.

38. Panchal N, Kamal R, Orgera K, et al. The Implications of COVID-19 for Mental Health and Substance Use. https://www.kff.org/reportsection/the-implications-of-covid-19-for-mental-health-andsubstance-use-issue-brief/: Kaiser Family Foundation; Aug 212020.

39. Torous J, Jan Myrick K, Rauseo-Ricupero N, et al. Digital Mental Health and COVID-19: Using Technology Today to Accelerate the Curve on Access and Quality Tomorrow. JMIR Ment Health. 2020; 7(3): e18848.

40. Lewis C, Gibson S, Bisson JI. Dropout from psychological therapies for post-traumatic stress disorder (PTSD) in adults: systematic review and meta-analysis, Eur J Psychotraumat, 2020; 11(1): 1709.

41. FDA U.S. Food and Drug Administration, 2018, online, https://www. accessdata.fda.gov/cdrh_docs/pdf18/K180173.pdf

42. Malowney M, Keltz S, Fischer D, et al. Availability of outpatient care from psychiatrists: a simulated-patient study in three US cities. Psychiatr Serv. 2015; 66(1): 94-96.

43. Mitchell C, McMillan B, Hagan T. Mental health help-seeking behaviours in young adults. Br J Gen Pract. 2017; 67(654): 8-9.

44. Pham Q Khatib Y, Stansfeld S, et al. Feasibility and efficacy of an mHealth game for managing anxiety: "Flowy" randomized controlled pilot trial and design evaluation, Fames Hlth, 2016; 5(1): 50-67.

45. Meuret AE, Rosenfield D, Seider A, et al. Respiratory and cognitive mediators of treatment change in panic disorder: Evidence for treatment specificity. J Consult Clin Psych. 2010; 78(5): 691-704. 